\title{
Evolving the Autosteering of a Car Featuring a Realistically Simulated Steering Response
}

\author{
Vsevolod Nikulin \\ Graduate School of Science and \\ Engineering, Doshisha University \\ 1-3 Tatara-miyakodani, Kyotanabe, \\ Kyoto 610-0321 \\ Japan \\ nikulin2016@sil.doshisha.ac.jp
}

\author{
Albert Podusenko \\ Graduate School of Science and \\ Engineering, Doshisha University \\ 1-3 Tatara-miyakodani, Kyotanabe, \\ Kyoto 610-0321 \\ Japan \\ podusenko2016@sil.doshisha.ac.jp
}

\author{
Ivan Tanev \\ Graduate School of Science and \\ Engineering, Doshisha University \\ 1-3 Tatara-miyakodani, Kyotanabe, \\ Kyoto 610-0321 \\ Japan \\ itanev@sil.doshisha.ac.jp
}

\author{
Katsunori Shimohara \\ Graduate School of Science and \\ Engineering, Doshisha University \\ 1-3 Tatara-miyakodani, Kyotanabe, \\ Kyoto 610-0321 \\ Japan \\ kshimoha@sil.doshisha.ac.jp
}

\begin{abstract}
We consider the area of intelligent road vehicles, especially, the topic of automated vehicles. Focusing on the importance of the automated steering, we address the challenge of automated keeping of a car in the middle of the driving lane. Our objective is to investigate the feasibility of employing genetic programming (GP) to evolve the automated steering of a car. The latter is implemented in the Open Source Racing Car Simulator (TORCS) with a realistically modeled steering featuring both a delay of response and a rate limit. We propose two approaches aimed at improving the efficiency of evolution via GP. In the first approach we implement an incremental evolution of the steering function by commencing the evolution with an ideal car and gradually increasing the degree of its realism (i.e., the amount of steering delay) in due course of evolution. The second approach is based on incorporating expert knowledge about the (expected) structure of the steering function according to the servo control model of steering. The experimental results verify that the proposed approaches yield an improved efficiency of evolution in that the obtained solutions are both of a better quality and could be obtained faster than those of the canonical GP.
\end{abstract}

Permission to make digital or hard copies of part or all of this work for personal or classroom use is granted without fee provided that copies are not made or distributed for profit or commercial advantage and that copies bear this notice and the full citation on the first page. Copyrights for third-party components of this work must be honored. For all other uses, contact the owner/author(s).

GECCO'18, July 15-19, 2018, Kyoto, Japan

(C) 2018 Copyright held by the owner/author(s).

ACM ISBN 978-1-4503-5618-3/18/07 \$15.00

DOI: $10.1145 / 3205455.3205547$

\section{CCS CONCEPTS}

Information systems $\rightarrow$ Process control systems

\section{KEYWORDS}

Genetic Programming, Automated Control, Software Agent, Autosteering

\section{INTRODUCTION}

Lane keeping could be viewed as a particular case of the wellstudied problem of path tracking. Also, lane keeping problem could be considered from the viewpoint of the behavior software agents situated in stochastic environments. The environmental information of such agents, as illustrated in Fig. 1, consist of the angle between longitudinal axis of the car main and direction of the lane $(\theta)$, distance between the center of the car and middle of a lane (e), speed of the car (V), current angular position of the steering (front) wheels $(\delta)$ and lateral acceleration of the car (a).

We consider the development of such steering control function that, for the currently perceived environmental information, results in such a steering angle that allows for an optimal lane keeping trajectory of the car. By optimal lane keeping trajectory we assume a simultaneous satisfaction of the following two conditions:

- Minimization of the mean absolute deviation of the center of the car from the middle of a lane, and

- Minimization of the mean absolute lateral acceleration. 


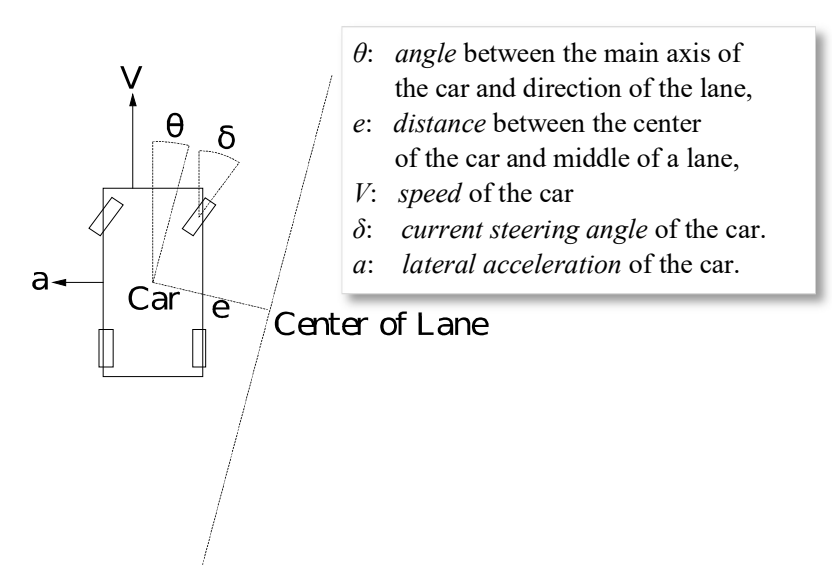

Figure 1: Perceived environmental information and the state of the car.

The first condition is intended to facilitate a steering behavior that keeps the car as close as possible to the center of the lane. The second condition is introduced in order to prevent oscillations of the car around the centerline. Such oscillations are pertinent to the feedback error-correcting control of the real-world cars due to the feedback latencies.

In order to combine the realism of the real-world cars with the safety, speed of prototyping and the low running costs of the simulated cars, in our research we use a model of the car and its environment, realistically simulated in the Open Source Racing Car Simulator (TORCS) [1]. Moreover, in an attempt to bridge further the reality gap between the real-world road cars and the car, simulated in TORCS, we enhanced the latter by introducing both (i) a nonlinearity of the steering system as a limit (of 30 degrees per second) of the rate of turning the front wheels of the car and (ii) a controllable steering delay (of up to $400 \mathrm{~ms}$ ).

The rate limit is a result of (i) the finite power of the hydraulic power-assisted steering systems of the modern road cars and (ii) the significant forces (caused by friction between the components of steering system, friction between the tires and the road surface, inertia of wheels, gyroscopic effect of rotating wheels, etc.) that resist the turning of the steering wheels.

Latencies in the control of steering are also an inevitable phenomenon in the steering systems of real-world road cars [2]. The overall steering delay is a cumulative result of the interplay of the delays in the steering components (gearbox, steering shaft, steering rack, knuckle arms, ball joints, bearings, etc.) either due to their elasticity (steering shaft, knuckle arms, etc.) or mechanical plays (bearings, ball joints, etc.). These delays are additionally aggravated by the elasticity of the sidewall of the (front) tires, which implies that the steering input applied to the wheels would not necessarily result in an instant turning of the tires of the car.

A more detailed description of the vehicle dynamics could be found in [2]. Without touching in details the underlying physics of the moving car, we would like to emphasize that taking into considerations the steering delays is very important for the development of adequate (stable) controller of the steering of the car.

With regard to the applicability of the proposed approach for a real autonomous lane-keeping car, the main issue will be acquiring sensors data. The way of extracting necessary information (namely - the lateral and angular position of the car with respect to the center of the lane) is beyond of the topic of our current. Hence, we assume that the required environmental information is already available, and focus on how an explicit modularization of the expected solution facilitates both the efficiency of evolution and the effectiveness of the latter.

Most of the existing machine learning approaches, applied for the automated development of steering, utilize an environmental information that inherently exhibits some "look-ahead" features. For example, the steering controller described in [3] decides the steering angle based on the image feed obtained from a video camera mounted in front of the car. We recognize that such approaches could facilitate the "anticipation" of future environmental situation(s) - and, moreover - that such anticipation is relevant for countering the detrimental effects of delayed, outdated steering feedback. However, we speculate that often in real-world situations, such looking-ahead perception information is not always accessible, for example, when driving in heavy traffic, poor visibility conditions (night, rain, snow, fog, etc.), or immediately behind a large vehicle (bus, truck, etc.). Therefore, we assume that constructing a delayed steering function based only on reliably obtainable environmental information pertinent to the immediate surroundings of the car would be an important task to solve in order to ensure an adequate anytime, all-weather steering control of the car.

The objective of our research is to evolve - by means of genetic programming (GP) - the optimal steering function of a car with realistically simulated steering response, based on realistic yet constrained, currently perceived perception information. The evolutionary development of the optimal steering function could be considered as an evolution of an algebraic function of the instantly perceived environmental variables.

Several related publications ([4-6]) are discussing a similar topic. However, in the current work we investigate whether introducing a domain-specific knowledge [7-8] into GP - used to evolve the optimal steering function - would facilitate either an improved efficiency of evolution or better quality of the obtained steering (or both). The introduced domain-specific knowledge is the (expected) form of the evolved steering function, inspired by the well-studied servo-control model of steering. The latter is recognized as a plausible model of the steering behavior of human drivers [9].

The remaining of the article is organized as follows. Section 2 describes the proposed approach of evolving the optimal steering function via GP. Section 3 presents the obtained experimental results. Finally, Section 4 draws a conclusion.

\section{PROPOSED APPROACH}

\subsection{Basic Concept}

We conducted our research on a car, modelled in the open source racing car simulator (TORCS). Advantages of the simulated car for researching the optimal control of vehicles is as mentioned in [10], and include: (i) the adequate level of realism of simulation of both the environment and dynamics of the car, (ii) crash-proof (software) implementation of the car, (iii) computational efficiency, and (iv) openness of its source code. In addition to these advantages, one of the features of TORCS, relevant to our 
Evolving the Autosteering of a Car with Realistically Simulated Steering Response

research, is the ability to model both the cars with variable steering delay, and ideal cars with instant steering response.

GP as a problem-solving paradigm inspired by natural evolution, is elaborated in [11]. In the considered case of evolving the steering function of the car the terminal set consists of the environment information. Because the objective of our research is to develop the functionality of the purely reactive steering agent (that decides and applies the optimal steering angle based on the currently perceived information about the state of the car and its surroundings), we implemented the internal state of the agent as a memory that stores the values of perception information pertinent to the previous instant of simulated time: $\theta, \mathrm{e}, \mathrm{V}$ together with their calculated (based on the difference between of the values of two consecutive instances) derivatives $\dot{\theta}, \dot{e}, \dot{V}$ and $\dot{a}$.

As a function set of GP, employed to evolve the algebraic function of the steering angle (as a parse tree) we use simple arithmetical operations $\{+,-, *, /\}$. The choice is based on the known form of the optimal steering function - according to the servo-control model - for the ideal car featuring an instant steering response. Detailed description of the parameters of GP is given in later section 3.1.

We propose a fitness function that reflects our intention to balance the satisfaction of previously mentioned two conditions of quality of steering - (i) minimal mean absolute deviation of the center of the car from the middle of a lane, and (ii) minimal mean absolute lateral acceleration. We calculate both the mean absolute deviation and mean absolute lateral acceleration during the test run of the car steered by the evaluated genetic program on a sample test track. A detailed description of both the fitness function and the track are given later in Section 3.2.

\subsection{Normalizing the Range of the Values of Evolved Steering Function}

The output of the function generated by GP (i.e., the action of the steering agent) should be adjusted to real values within the range between -1 to 1 (corresponding to the full steering lock to the left and right, respectively) in order to comply with the acceptable range of steering commands as defined in TORCS. Zero value correspond to the position of the steering wheels aligned with the longitudinal axis of the car. In order to map the potentially unconstrained range of values $\delta$ of the evolved steering functions into $\delta_{\text {adjusted }}$ in the range of between -1 to 1 , we applied the normalizing function as shown in Equation (1).

$$
\delta_{\text {adjusted }}=-1+\frac{2}{1+\frac{1}{2^{\delta}}}
$$

\subsection{Incremental GP}

The major novelty in the proposed approach is the introduction of gradually increasing (in due course of evolution) of the steering delay of the car. Because the steering delay is not constant, but rather - a variable that increases in due course of evolution, we introduce the current value of steering delay as an additional
GECCO’18, July 15-19, 2018, Kyoto, Japan

terminal in the set of terminal symbols. We denote this terminal as $\Delta t$. The flowchart of the proposed approach is depicted in Fig. 2.

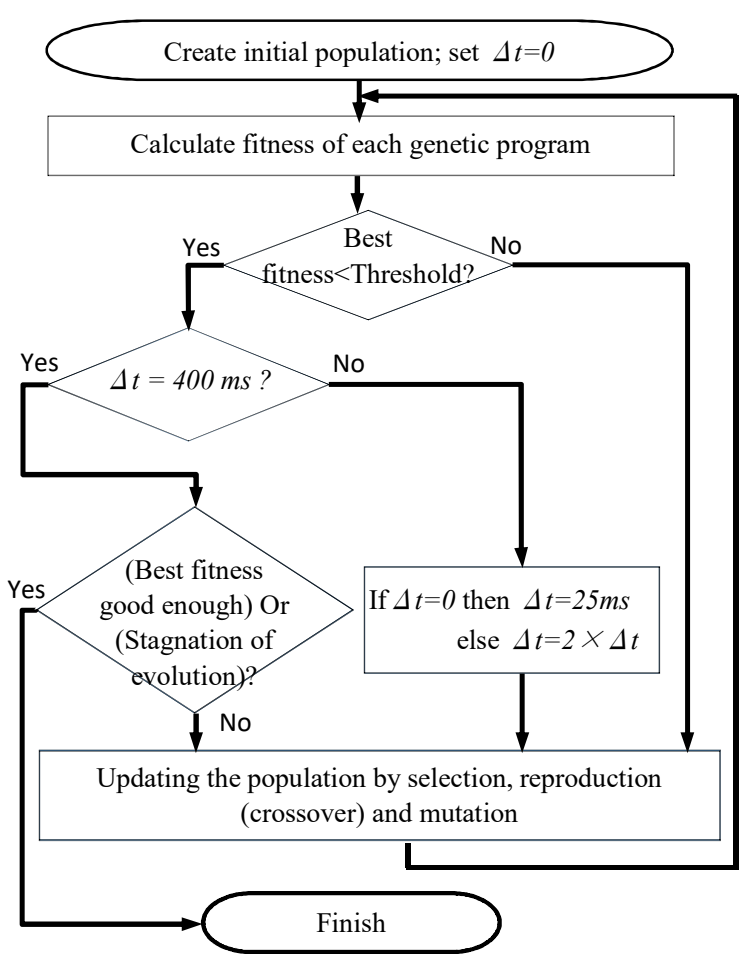

Figure 2: Flowchart of the proposed incremental genetic programming approach.

Our proposal to employ an incremental evolution is motivated by our observations that canonical GP (in which the parameters of the modelled car, including its steering delay are kept constant in due course of evolution, e.g., either $400 \mathrm{~ms}$ in case of a realistically modelled car, or $0 \mathrm{~ms}$, in case of an ideal one) is able to easily evolve a good quality steering function for an ideal car, while, on the realistically modelled car featuring a steering delay, evolving a similarly good steering function becomes impossible. Moreover, we hypothesize that the steering functions that control well the realistically modelled car might contain (genetic) fragments that are common with the steering functions for ideal car. Incremental evolution would facilitate an improvement of the efficiency of evolution by allowing the evolved functions that control the car with (increasingly) delayed response to inherit these fragments from the optimal steering functions of the ideal car.

In the proposed implementation of incremental GP, we start the evolution with an ideal car $(\Delta t=0)$ and in due course of evolution - when the best evolved steering function of the current generation reaches some threshold value of quality of control (fitness) - we gradually increase the steering delay of the car. We increase the delay $\Delta t$ from 0 to $25 \mathrm{~ms}, 50 \mathrm{~ms}, 100 \mathrm{~ms}, 200 \mathrm{~ms}$, and, finally, to $400 \mathrm{~ms}$. The latter value corresponds to the typical 
steering delay of the real world cars, and, therefore, a good fitness value obtained for a car with such a steering delay would indicate a good quality of steering of a realistically simulated car.

\subsection{The Form of Solution According to the Servo Control Model}

One of the most widely used models for car with non-delayed steering is the servo control model [12], in which the steering is seen as an error-correcting behavior that continuously attempts to minimize both the lateral and angular deviations of the car from the center of the lane $(\mathrm{CoL})$. According to this model, the steering angle $\delta$ is calculated from the following Equation (2):

$$
\delta=k_{1} e+k_{2} \theta
$$

Indeed, according to our preliminary experimental results, this equation provides a stable and consistent driving around a set of given test tracks. Varying the parameters $k_{1}$ and $k_{2}$ may result in different trade-off between the maximum deviation from the center of the lane at the entry of a corner, average lateral deviation during steady state cornering, rate of returning to the center of the lane at the exit of a corner, and severity of oscillations (if any) at the entry and exit of corners. However, the function expressed in Equation (2), yet providing an adequate steering control of an ideal car, could not be applied for the steering of cars featuring a non-zero steering delays. Consonant with the feedback control theory, and, especially, with the Nyquist stability criterion, a steering delay of, say, just $200 \mathrm{~ms}$, results in instability of the control even on strait sections of the road. This instability is manifested by the oscillating values of the steering angle, angular and lateral deviations from the center of the lane. More detailed discussion about the stability of feedback systems with a time lag could be found in [13].

Having a time lag, we wish to have a way of prediction for future perceptions. In an ideal case, knowing exact information about future, it is possible to develop a steering function as if control was not delayed. This prediction can be built using dynamics of change in perception information. Hence, we need to find a way to predict future values of $e$ and $\theta$, then we can use servo control model for control. The idea of GP enhancement proposed in this section is as follows: evolve not the function of control itself, but a way of prediction future perception information. The form of the evolved steering function in canonical GP and in the proposed approach is depicted in Fig. 3.

As Fig. 3 illustrates, instead of evolving an genetic program (individual) that is represented by a single parse tree, as in canonical GP (Fig. 3, top), we evolve genetic program that comprises a forest of five trees. Each of these trees encode a separate component of the servo-control model: two of these subtrees represent constants; another two encode the anticipated changes in $e$ and $\theta$ in the immediate future, after the time equal to the steering delay, and the remaining one is just a "free" component for consistency. The evolution of these parse trees is strongly typed in that the crossovers operation is allowed only between the trees that represent an identical component of the servo control model.

By introducing such a domain specific knowledge into GP we intend to reduce the presumably huge search space of the canonical GP and to explore only such areas in the landscape that conform to the servo-control model of steering.

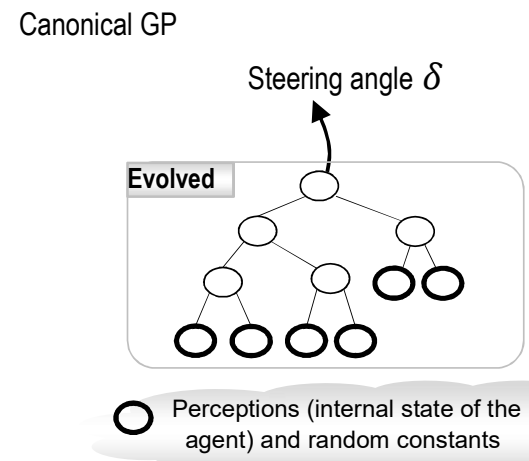

Proposed Approach: GP for evolution of servo control model

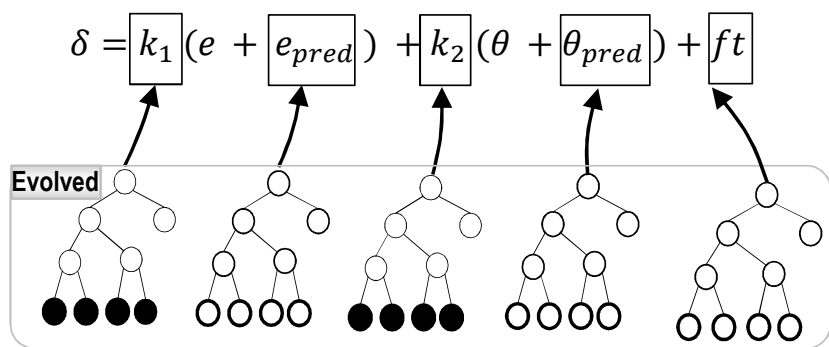

Random constants

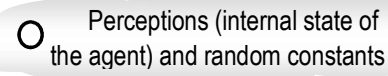

Figure 3: The forms of evolved steering functions in canonical GP (top) and the proposed GP for evolution of servo control model with anticipation (bottom).

\section{EXPERIMENTAL RESULTS}

\subsection{Experimental Setup}

A snapshot of the simulated car in TORCS and a list of its main features are shown in Fig. 4 and Table 1, respectively. The trial runs during the evaluation of the fitness of evolved steering functions were performed on a sample track as shown in Fig. 5.

During the trial run the car starts to accelerate on the straight part of the track without steering control and after reaching the desired speed, this speed keeps constant via simulated cruise control, and the evolved steering function takes control of the car. The chosen constant speed of the car $(50 \mathrm{~km} / \mathrm{h})$ corresponds to a comfort driving and guarantees that the turning forces stay well below the available friction forces even in the sharpest turn of the track. The car starts its initial acceleration 3 meters away from the center of a lane in order to cut off the solutions which cannot 
Evolving the Autosteering of a Car with Realistically Simulated Steering Response

bring car to the center of the lane from the very beginning of the trial.

If the car is unable to arrive at the finish of the track (e.g. if it runs off the track), the steering function that controls the car is penalized by fitness that is set a high value, corresponding to a very low quality of driving. Represented track includes a combination of both straight- and curved sections that are intended to test the ability of the evolved steering to control the car robustly in diverse layouts of the road.

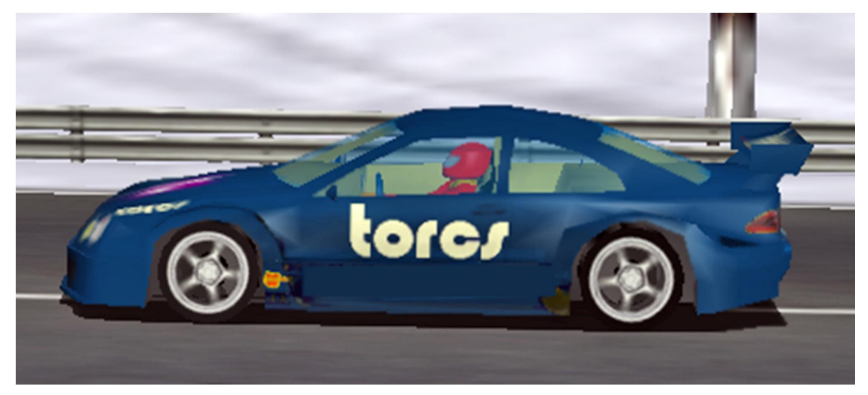

Figure 4: Snapshot of the simulated car in TORCS

Table 1: Features of the simulated car

\begin{tabular}{lc}
\hline Feature & Value \\
\hline Model & CLK DTM \\
Length, m & 4.76 \\
Width, m & 1.96 \\
Height, m & 1.17 \\
Mass, kg & 1050 \\
Front/rear weight repartition & $0.5 / 0.5$ \\
Height of center of gravity, $\mathrm{m}$ & 0.25 \\
Coefficient of friction of tires & 1.2 \\
Steering delay, ms & 400 \\
Drivetrain & Front engine, rear wheels drive \\
\hline
\end{tabular}

Table 2: Main Parameters of GP

\begin{tabular}{|c|c|}
\hline Category & Value \\
\hline $\begin{array}{l}\text { Genetic } \\
\text { representation }\end{array}$ & XML-DOM parse tree \\
\hline Function set & Algebraic functions: $\{+,-, /, *\}$ \\
\hline Terminal set & $\begin{array}{l}\text { Parameters of environment information } \\
\text { and their derivatives }\end{array}$ \\
\hline Population size & 400 individuals \\
\hline Selection & Binary tournament, ratio 0.1 \\
\hline Elitism & Best 8 individuals \\
\hline Mutation & $\begin{array}{l}\text { Random subtree mutation; ratio: } 0.05 \text {, } \\
\text { growing with stagnation }\end{array}$ \\
\hline Fitness value & $\begin{array}{l}\text { Mean absolute deviation from a center of } \\
\text { lane }+ \text { Mean absolute lateral acceleration }\end{array}$ \\
\hline $\begin{array}{l}\text { Termination } \\
\text { criteria }\end{array}$ & $\begin{array}{l}\# \text { Generations }=200 \\
\text { or stagnation of fitness for } 32 \text { generations }\end{array}$ \\
\hline
\end{tabular}

GECCO’18, July 15-19, 2018, Kyoto, Japan

The main parameters of the GP are depicted in Table 2. These parameters do not include incremental aspect of the proposed approach, which is described in Fig. 2.

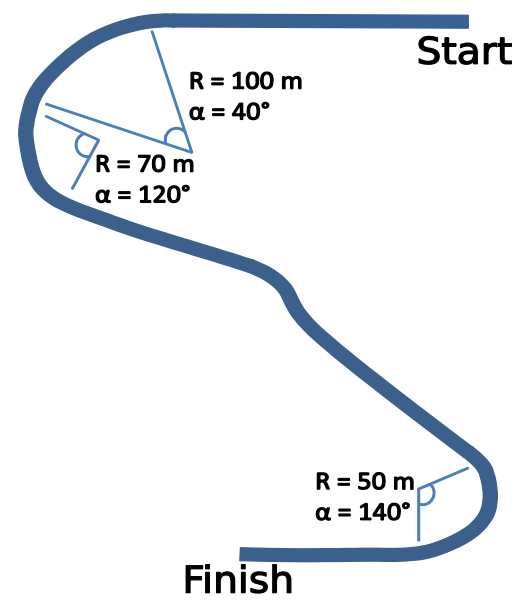

Figure 5: The sample track used for evaluating the quality of evolved steering control of the car.

\subsection{Evaluation of the driving quality}

Fitness function for the proposed GP is calculated based on the behavior of the car on a sample track. For estimating this value for each individual, we run the simulated car on the same sample track in the identical conditions. The fitness function, as a measure of quality of steering control of the car, is designed based on environmental information at each sampling period (frame) of simulation as shown in Equation (3) :

$$
F=\frac{\sum_{i=0}^{N}\left|e_{i}\right|}{L}+C \frac{\sum_{i=0}^{N}\left|a_{i}\right|}{L}
$$

Where $L$ is a total length of the track, $e_{i}$ and $a_{i}$ are the lateral deviation (i.e., the distance between the center of the car and middle of the lane) and lateral acceleration of the car at $i$ th frame, respectively. Better quality of steering would results in lower values of the two additive terms of the fitness function, and lower overall value of the latter. First term in this expression represents the first condition of optimality: our desire to drive the car as close as possible to the center of a lane. The second term reflects our intention to prevent an oscillating steering behavior of the car. The minimized steering oscillations would be manifested by minimized mean absolute value of the lateral acceleration. In addition, the second term is scaled down by constant coefficient $C$. This coefficient can be varied to balance the contribution of those two terms to the overall quality of steering of the car. In our experiments $C=0.25$. 


\subsection{Experimental Results}

At this section we will compare the fitness convergence characteristics of GP in three experimental cases:

1. Evolving the steering function of a realistically simulated car with steering delay $\Delta \mathrm{t}=400 \mathrm{~ms}$ with canonical GP without enhancements,

2. Evolving the steering function of a realistically simulated car with steering delay $\Delta \mathrm{t}=400 \mathrm{~ms}$ via incremental GP without specific form of the solution, and

3. Evolving the steering function of a realistically simulated car with steering delay $\Delta \mathrm{t}=400 \mathrm{~ms}$ via incremental GP with servo control form of the solution.

Evolution has been implemented using XML-based genetic programming framework described in [13]. The experimental results are shown in Figure 6.
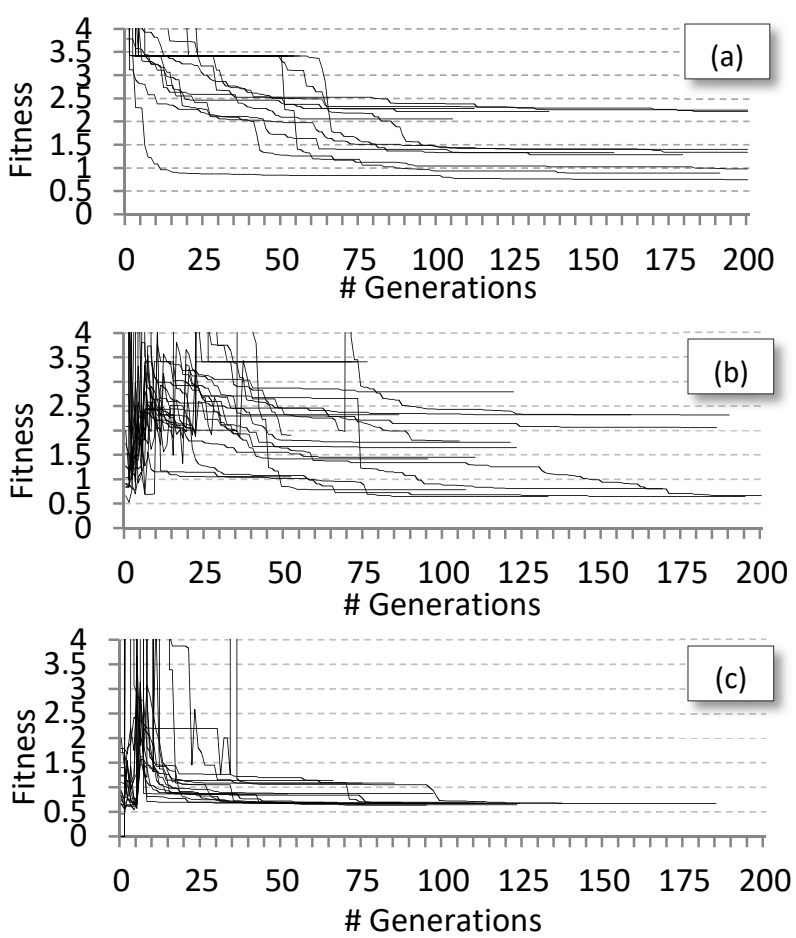

Figure 6: Experimental results: fitness convergence of 20 runs of canonical GP without enhancements (a), fitness convergence of incremental GP without specific form of the solution (b), and fitness convergence of incremental GP with servo control form of the solution (c)

Quantitative comparison is shown in Table 3. While introducing incremental approach allows evolution to find much better solution, introducing servo control model of the solution drastically simplifies the fitness landscape, which results in more robust and stable convergence (it could be seen in reducing of standard deviation of the best fitness value of sample GP run). Even the worst fitness improved, which also signalizes of improved robustness.

Another comparison between approaches represented in Fig. 7: dynamic of change of deviation from the center of the lane in time during the test run for best solutions. The worst is the result found by canonical GP; both conditions of optimality are violated: large mean deviation and presence of oscillations. On the other hand, solutions of plain incremental GP and incremental GP enhanced with specific form of the solution have similar fitness value, but different driving style. In the second case, overall deviation is smaller; software agent tries to keep the car to the lane as close as possible, suffering from oscillations. In the first case, the situation is opposite: larger deviation, but smaller oscillations. However, this altering in driving styles is not a specific feature of the approaches. It is just different sub-optimal strategies found by evolution and not a characteristic difference between methods.
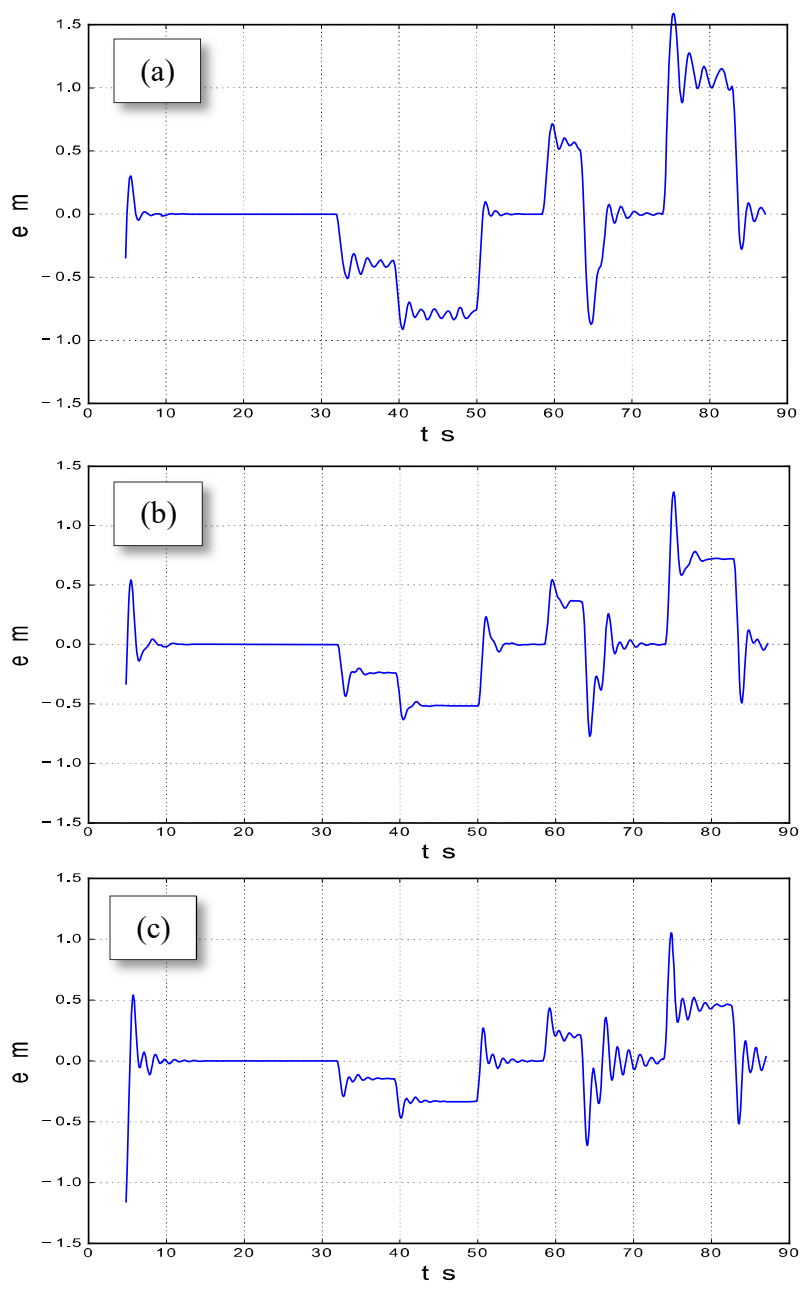

Figure 7: Experimental results: deviation from the center of the lane during the test run of the best solution of canonical GP (a), deviation from the center of the lane during the test run of the best solution of incremental GP (b), and deviation from the center of the lane during the test run of the best solution of incremental GP with servo control form of the solution (c) 
Table 3: Characteristics of convergence of all compared approaches for GP

\begin{tabular}{|c|c|c|c|}
\hline \multirow[b]{2}{*}{ Feature } & \multicolumn{3}{|c|}{ Approach } \\
\hline & Canonical GP & Incremental GP & $\begin{array}{c}\text { Incremental } \\
\text { GP with } \\
\text { Servo Model }\end{array}$ \\
\hline Min (best) fitness & 0.74 & 0.65 & 0.62 \\
\hline $\begin{array}{l}\text { Max (worst) } \\
\text { fitness }\end{array}$ & 3.41 & 3.41 & 1.13 \\
\hline Average fitness & 2.32 & 1.81 & 0.76 \\
\hline $\begin{array}{l}\text { Standard } \\
\text { deviation }\end{array}$ & 1.02 & 0.93 & 0.16 \\
\hline $\begin{array}{l}\text { \#Fitness cases } \\
\text { within }[0.5-0.75]\end{array}$ & 1 (of 20) & $3($ of 20$)$ & 15 (of 20) \\
\hline
\end{tabular}

Finally, algebraic expression (in simplified form) for a sample best-of-run steering solution, obtained via canonical GP on the car with steering delay of $400 \mathrm{~ms}$, usually is hard to comprehend, as illustrated in Equation (3).

$$
\delta=\frac{2 \dot{\theta}-\dot{e}-\theta-e}{\delta+\frac{3 \dot{a} \theta}{\delta+3}+\frac{v}{\left(\frac{\dot{a}}{8}+\frac{\theta}{8}\right)\left(\frac{9 \dot{a}}{8}+\frac{\dot{e}}{8}\right)-3}+5-\frac{\delta+\dot{e}-a-4}{2 \Delta t-v}+\frac{e+3}{3 \delta^{2}}}
$$

However, a sample best-of-run steering solution obtained via incremental GP on a car with incrementally increased steering delay (up to $400 \mathrm{~ms}$ ), shown in Equation (4), is much more elegant and comprehensible.

$$
\delta=\frac{36 \theta}{5 v}+\theta+\frac{2 \dot{\theta}+2 \theta-e}{\frac{1}{5 v}-\theta+4}
$$

Where $v$ is the constant speed $(13.8 \mathrm{~m} / \mathrm{s}$ corresponding to 50 $\mathrm{km} / \mathrm{h}$ ), and the angular deviation from the center of the lane $\theta$ (in radians) is small compared to 4 in the denominator. Therefore, this steering solution could be seen as a modification of the servo control model, which uses derivative of $\theta$ to predict the future position of the car. This proves our assumption about form of the solution.

The main difference in the last proposed enhancement is that the servo control form of the solution is explicit. Nevertheless, sometimes in the process of evolution, tricky expressions come up For example, in Equation (5), subexpressions, which are intended to be anticipated changes in $\theta$ and $e$ in the immediate future, are mutually subtracted almost to 0 . All anticipation comes in the "free" component. However, it is still a variation around servo control model.

$$
\delta=1.999(\theta+a)-1.977(e+a)+\frac{\dot{\theta}}{8 \Delta t^{2}}
$$

\section{CONCLUSION}

In summary, we have implemented several enhancements over canonical Genetic Programming approach for deriving control function of a realistically simulated car. We showed that both proposed technics lead to improvement of the convergence of sample GP run. Gradual increment of the delay in the control corresponds to gradual complication of the problem during evolution, which, in turn, gives us convergence to the desired function. Explicitly giving solution specific form of servo control model significantly reduces the size of fitness landscape resulting in convergence that is more robust. Both enhancements correspond to different way of using domain specific knowledge.

Developing a steering function for realistically simulated car is a hard problem, which requires taking into account many details. It is nearly impossible to derive a complete model of the car's mechanics. However, we have knowledge about simplified models; we are able to make right assumptions and approximations. Having this domain-specific knowledge, we are able to propose proper guidance for the artificial evolution making it converge much faster and robust than its unguided analog.

\section{REFERENCES}

[1] Wymann B, Dimitrakakis C, Sumner A, Espí e E, Guionneau C, Coulom R 2013, TORCS, the open racing car simulator, v1.3.5. http://www.torcs.org

[2] Gillespie T. 1992. Fundamentals of Vehicle Dynamics. Premiere Series Bks, Society of Automotive Engineers

[3] Bojarski M, Testa DD, Dworakowski D, Firner B, Flepp B, Goyal P, Jackel LD, Monfort M, Muller U, Zhang J, Zhang X, Zhao J, Zieba K 2016. End to end learning for self-driving cars. CoRR abs/1604.07316,

[4] Nikulin V, Podusenko A, Tanev I, and Shimohara K 2017. On the Effects of Anticipatory Modeling on Quality of Automated Steering of Simulated Car. In Proceedings in FAST-zero 2017, 6 pages

[5] Nikulin V, Podusenko A, Tanev I, and Shimohara K 2017. Evolving the Autosteering of a Car with Realistically Simulated Steering Response. In Proceedings in SICE 2017, 6 pages

[6] Nikulin V, Podusenko A, Tanev I, and Shimohara K 2017. Regression-based Model for Autosteering of a Car with Delayed Steering Response. In Proceedings in 2017 IEEE International Conference on Data Science and Advanced Analytics (IEEE DSAA), pages 412-419

[7] Angeline P J 1994, Genetic Programming and Emergent Intelligence. Advances in Genetic Programming, pp 75-98.

[8] Passone S, Chung PWH, Nassehi V 2006. Incorporating domain-specific knowledge into a genetic algorithm to implement case-based reasoning adaptation. Knowledge-Based Systems, vol. 19, pp 192-201

[9] Cacciabue PC 2007. Modelling Driver Behaviour in Automotive Environments: Critical Issues in Driver Interactions with Intelligent Transport Systems. Springer-Verlag New York, pp 91-93.

[10] Sharma DG, Tanev I, Shimohara K 2016, Automatic Classification of Driving Conditions for the Detection of Driver-Induced Steering Oscillation. In: Proceedings of The Second International Conference on Electronics and Software Science (ICESS2016), pp 88-95.

[11] Koza JR, Keane MA, Streeter MJ, Mydlowec W, Yu J, Lanza G 2003, Genetic Programming IV: Routine Human-Competitive Machine Intelligence. Kluwer Academic Publishers.

[12] McRuer DT, Allen RW, Weir DH, Klein RH 1977. New results in driver steering control models. Human Factors 19(4), pp 381-397

[13] Krall MA 1964. Stability Criteria for Feedback Systems with a Time Lag. Journal of the Society for Industrial and Applied Mathematics Series A Control, 2(2), pp. 160-170.

[14] Tanev I, Shimohara K 2010, Xml-based genetic programming framework: design philosophy, implementation, and applications. Artificial Life and Robotics 15(4):376-380 